\title{
Epilepsy in horses: Aetiological classification and predictive factors
}

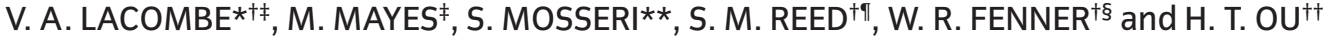 \\ ${ }^{\dagger}$ Department of Veterinary Clinical Sciences, College of Veterinary Medicine, The Ohio State University, Columbus, USA \\ ${ }^{\ddagger}$ College of Pharmacy, The Ohio State University, Columbus, USA \\ $\S$ MedVet Medical Center, Worthington, Ohio, USA \\ "Rood \& Riddle Equine Hospital, Lexington, Kentucky, USA \\ **The Equine Clinic, Peyrolles, France \\ ${ }^{\dagger+}$ The University of Michigan College of Pharmacy, Ann Arbor, USA
}

*Correspondence email: lacombe.2@osu.edu; Received: 10.05.11; Accepted: 08.10.11

\begin{abstract}
Summary
Reasons for performing the study: In contrast with reports in man and small animals, a systematic classification of seizures in horses is lacking. Objectives: The purpose of this study was to classify seizures based on their aetiology and to characterise epilepsy in 104 horses presented for seizures at the Ohio State University Veterinary Medical Center between 1988 and 2009.

Methods: In a retrospective observational study, seizures were classified by aetiology based on history, clinical observations, diagnostic investigations (e.g. electroencephalograms, cerebrospinal fluid and computed tomography imaging of the head) and post mortem examinations, when available. Univariate and multivariate logistic regression analyses were performed.

Results: Epilepsy (i.e. 2 or more recurrent seizures) was identified in 70\% of cases, and further classified as symptomatic (i.e. structural brain pathology, $35.6 \%$ of cases), cryptogenic (i.e. unknown, $54.8 \%$ of cases) and idiopathic (i.e. suspected genetic predisposition, $2.7 \%$ of cases). Normal neurological examination on admission, the presence of seizures unprovoked by any identified factors and paroxysmal epileptiform activity on electroencephalogram recordings were all strongly $(P<0.05)$ correlated with epilepsy on univariate analysis. For a horse with generalised seizures, the odds of having epilepsy was 7 times lower compared with a similar horse with partial seizures $(\mathrm{P}<0.05)$ in multivariate modelling.

Conclusions: Seizure aetiology was symptomatic or cryptogenic in most horses, whereas reactive seizures and idiopathic epilepsy were less common.

Potential relevance: This study is the first attempt to classify seizures and to characterise epilepsy in a referral-based equine population. Predictive factors of epilepsy in horses were similar to those reported in other species and may assist the clinician with the early diagnosis of epilepsy.
\end{abstract}

Keywords: horse; seizures; epilepsy; idiopathic; cryptogenic; symptomatic

\section{Introduction}

Epilepsy is a chronic neurological condition characterised by recurrent seizures, according to the International League Against Epilepsy (ILAE) [1-3]. Epilepsy is the most common neurological disorder in dogs, and epilepsy classification has been attempted in dogs and cats [4-9]. In contrast with studies conducted in small animals and man, a systematic description of seizures in mature horses is lacking, and epilepsy has been rarely documented except during genetic disorders such as idiopathic juvenile Arabian and lavender foal syndrome $[10,11]$. As a result, a variety of terms have been used to describe equine seizure disorders in the literature, and little common agreement exists on the definition of epilepsy. Therefore, a common terminology for seizures and epilepsy is critically needed in horses and could help clinicians in the establishment of a diagnosis, which is often challenging, in part because of the small number of tools capable of imaging the equine brain. Although predictive factors of epileptic seizures have been reported in dogs and man in order to assist in the early diagnosis of epilepsy [5,12-14], similar study in horses is lacking. Therefore, the objectives of the present study were: 1) to classify seizures and epilepsy according to most recent accepted definitions in both human and small animal epileptology; and 2) to determine predictive factors of recurrent seizures in order to identify clinical characteristics of horses with epilepsy.

\section{Materials and methods}

\section{Case selection, inclusion criteria}

Medical records of 104 horses (all ages, except neonates aged <3 weeks) presented for seizure disorders between January 1988 and May 2009 at the Ohio State University Veterinary Medical Center were reviewed. Evidence of seizures was identified based on historical information, follow-up telephone communications with referring veterinarians (when available) and/or evaluation of seizures by at least one clinician when they occurred during hospitalisation.

\section{Procedures}

Case history, possible familial predisposition, concurrent diseases and history of seizures were reviewed. When available, circumstances of apparition (e.g. traumatic event), duration and frequency of seizures, and recent behavioural changes were recorded from the history. All horses underwent complete general physical and neurological examination on presentation. Complete blood count and serum biochemistry profile were also obtained. Electroencephalography (EEG) was performed on 63 horses (56 under general anaesthesia and 7 standing under chemical restraint), as previously described [15]. Briefly, analogue EEG recordings were performed on one of two 10-channel machines (Grass model $8^{\mathrm{a}}$ and Neurofax ${ }^{b}$ ) with 2 different types of montages (a bipolar left-to-right, back-to-front montage and a bipolar circular montage) by means of platinum subdermal needle electrodes placed in the scalp on the frontal, occipital and parietal areas. Visual analysis was performed on all recordings by board-certified neurologists. Computed tomography (CT) imaging of the head was performed in 40 anaesthetised horses by board-certified radiologists using a fourth generation single-slice helical CT scanner (Picker PQS) ${ }^{c}$, as previously described [16]. Other ancillary diagnostic tests included cerebrospinal fluid (CSF) analysis $(n=84)$ from the atlanto-occipital space in $87.5 \%$ of the cases, and was considered within normal limits if clear and colourless with $<1 \mathrm{~g} / \mathrm{l}$ of protein and $<5 \times 10^{6}$ white blood cells/l. Western blot analysis for equine protozoal myeloencephalitis (EPM) on both CSF and blood, and blood antibody titres or polymerase chain reaction (PCR) analysis for equine herpes virus myeloencephalopathy and West Nile Virus encephalitis were performed when appropriate. Skull radiographs were performed when indicated (e.g. head trauma). Post mortem examinations of the central nervous system, which includes gross and histopathological evaluation of section of the brain, brainstem and spinal cord, were performed in 30 horses by board certified pathologists.

\section{Classification of seizures}

Seizures were classified according to revised consensus of the ILAE and based on veterinary epileptological studies $[1,3,4,7,17]$. Seizures were 
classified according to their frequency $(<2$ and $\geq 2$ episodes), which included all of the episodes reported before referral and during hospitalisation. When seizures occurred repetitively within $24 \mathrm{~h}$, this cluster of seizures was considered as a single episode [1]. Seizures were further classified according to their aetiology (Axis 3 of the ILAE diagnostic scheme), based on the results of complementary diagnostic tests and post mortem examination (when available). Within each category, seizures were divided into partial or generalised. Seizures that involve a focal area of the cerebral cortex are classified as partial seizures and result in localised motor signs or sensations. Generalised seizures, which involve the entire cerebral cortex, result in generalised bilateral motor activity over the whole body, and can originate from both cerebral hemispheres from the onset (primary generalised seizures) or progress from partial seizure (secondary generalised seizures). Reactive seizures were identified because of the presence of a temporary systemic disease with a normal brain function. Epilepsy was defined as the recurrence of 2 or more seizures [4] and was further classified as symptomatic, cryptogenic, idiopathic, as follows: symptomatic epilepsy was diagnosed if a structural brain disorder was identified based on abnormal results of the neurodiagnostic tests (i.e. CSF analysis, CT scan and/or post mortem examination); cryptogenic (i.e. unknown) epilepsy was diagnosed if recurrent seizures were present in the face of normal results of the diagnostic tests (i.e. laboratory profile, CSF analysis, CT scan and/or post mortem examination within normal limits); and a diagnosis of 'idiopathic epilepsy' was made when no underlying structural brain or metabolic abnormality could be identified based on normal results of the diagnostic tests and a suspected genetic predisposition (i.e. Arabian horses aged $<1$ year).

\section{Data analysis}

Clinical data analysed to characterise epilepsy included: breed, sex, age, duration of neurological signs, history of head trauma, mentation, behaviour, cranial nerve and gait deficits, type of seizures, provoking factors, occurrence of seizures during hospitalisation, blood work analysis, EEG, CSF analysis, CT imaging of the head, skull radiographs, and post mortem examination. To facilitate logistic regression, continuous variables (e.g. age) were categorised by cut-off values based on distribution within a group. The significance of univariate associations was determined by the use of univariate logistic regression analysis. In order to account for potential confounding variables, a multivariate logistic regression analysis was developed on the basis of results for the univariate analysis. A critical P value of 0.05 was used as a criterion for inclusion of the variables in the multivariate model. Adjustment was made for data heteroscedasticity, using White-Huber robust standard errors [18]. All variables chosen for the multivariate logistic regression analysis were also analysed to determine if any interaction of dependency among variables occurred, by using a Pearson correlation test, which did not show any strong (i.e. <0.5) correlation between the chosen variables. Odds ratios (OR) were used to measure the association between each independent variable and the outcome of interest, and 95\% confidence intervals (95\% Cl) were calculated. $P$ values $\leq 0.05$ were considered as statistically significant. Data analysis was conducted using SAS software (version 9.2) ${ }^{d}$.

\section{Results}

\section{Description of the population}

The study population consisted of 19 males, 43 geldings and 42 females. Fourteen different breeds were represented, including Quarter Horses and related $(n=29)$, Thoroughbreds $(n=23)$, Arabians and crossed Arabians $(n=20)$, Standardbreds $(n=7)$ and various other breeds $(n=25)$. Age ranged from one month to 30 years, with a mean \pm s.d. of $10.3 \pm 6.6$ years. Eighty-eight $(84.6 \%)$ horses presented with a primary complaint of seizure-like activity (including abnormal behaviour) and 16 (15.4\%) horses only developed seizures during hospitalisation. Neurological examination abnormalities on admission and/or during hospitalisation were reported in 71 cases and included: abnormal behaviour $(n=23)$, cranial nerve deficits $(n=24)$, abnormal mentation (depression, hypersensitivity, agitation, disorientation in 41 cases) and gait deficits $(n=67)$. Seizures were defined as partial in 44 cases, and primarily and secondary generalised in 60 cases. Generalised seizures were characterised by tonico-clonic motor activity, with eye globe deviation, nystagmus or generalised rigidity. For horses affected with partial seizures, motor abnormalities involved areas of the face (e.g. muzzle or lip twitching/tremor, repetitive manic biting at the ground, or compulsive chewing/licking) or the body (e.g. compulsive kicking, tremors, lifting one limb, violent headshaking). In horses with suspected underlying brain diseases, 29 horses exhibited a single episode of seizure and epilepsy (i.e. recurrence of $\geq 2$ seizures) was identified in 73 cases (Fig 1).

Electroencephalographic recording demonstrated evidence of epileptiform activities, characterised by spikes, sharp waves, and spike and wave discharges in $28 / 52(54 \%)$ horses with recurrent seizures. Diffuse paroxysmal activity with high frequency and low amplitude discharges was recorded in 12/52 (23\%) additional horses with recurrent seizures. Sixteen horses underwent both EEG and cranial CT examinations, and EEG recording demonstrated abnormal cerebral electrical activity (e.g. epileptiform activities and/or diffuse paroxysmal activity) in 11 (69\%) horses, which had normal CT imaging of the head. Electroencephalography was within normal limits in 7 horses despite evidence of seizures in these horses. Electroencephalography remained inconclusive due to muscle artefacts in standing horses or due to chemical- or age-induced alterations in background pattern in 11 cases (6 of which had recurrent seizures). The CSF analysis was abnormal in $17 / 63$ (26\%) horses suffering from recurrent seizures and in 8/21 (33\%) horses exhibiting a single episode; abnormalities included neutrophilic pleiocytosis ( $n=7)$, evidence of previous haemorrhage $(n=2)$, lymphocytic pleiocytosis $(n=2)$, albuminocytological dissociation $(n=9)$ and combined abnormalities $(n=5)$. For horses

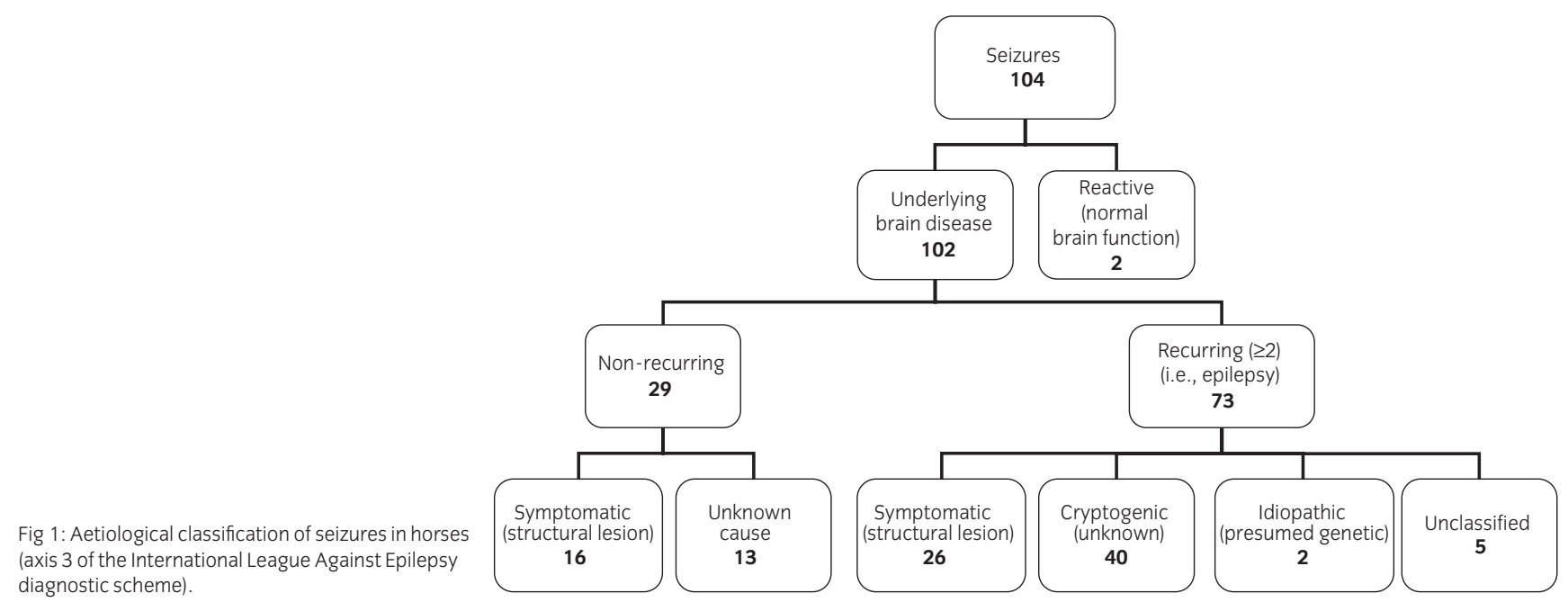

diagnostic scheme). 
demonstrating albuminocytological dissociation on CSF analysis, it was not possible to know if the elevation of total protein content was a cause or a consequence of the seizures because CSF was sampled within $48 \mathrm{~h}$ following an episode in the majority of cases. Western blot analysis for EPM was positive in the CSF of 5 cases. Computed tomography imaging of the head was unremarkable in $25 / 28(83 \%)$ of horses with recurrent seizures. In contrast, CT imaging of the head was abnormal in $7 / 12$ (53\%) horses exhibiting a single episode. Computed tomography abnormalities included traumatic intracranial lesions ( $n=4)$, space occupying lesions $(n=4)$, diffuse cerebral oedema $(n=1)$ and nasal mass invading the cribiform plate $(n=1)$. Post mortem examination of the brain was performed in 3 cases with CT abnormalities and confirmed the CT findings in all cases. One of the lesions could only be identified by contrast enhancement and was consistent with vascular event on post mortem examination.

Post mortem examination was abnormal in 82\% (9/11) horses exhibiting a single seizure episode, and revealed head trauma with multiple calvarial fractures and/or intracranial haemorrhage, vasculitis, meningitis, nasal adenocarcinoma with intracranial extension and intracranial vascular events. Post mortem examination was abnormal in 14/19 (73.6\%) horses exhibiting recurrent seizures, and revealed chronic underlying lesions, including congenital abnormalities and degenerative processes (white matter cavitation and focal neuronal dysplasia), cholesterol granulomas $(n=4)$, and neoplasia, which included pituitary adenomas $(n=1)$, lymphoma $(n=1)$ and oligodendrioma $(n=1)$. Post mortem examination remained inconclusive in 3 cases ( 2 of which had recurrent seizures).

\section{Aetiological classification}

Reactive seizures were recognised secondary to liver failure and to severe acute systemic haemorrhage in 2 horses (Fig 1). In horses exhibiting a single seizure episode, an underlying structural brain lesion (i.e. symptomatic seizure) was confirmed or suspected in 16 horses and the seizures were directly caused by an acute head trauma in 6/16 (37.5\%) of these horses. Diagnostic procedures were unremarkable, partial or inconclusive in 13 additional cases, leading to classification of seizures of unknown origin. In horses exhibiting recurrent seizures, epilepsy was classified as symptomatic in 26/73 (35.6\%) cases and as cryptogenic in 40/73 (54.8\%) horses. Five horses presented recurrent seizures but remained unclassified due to inadequate work-up. Based on early onset (age 4-5 months), breed predisposition (Arabian), unremarkable CSF analysis and CT imaging of the head, and good response to anti-epileptic therapy, 2 foals were diagnosed with idiopathic epilepsy.

\section{Clinical factors associated with epilepsy}

Based on the univariate logistic analysis, no association between age, breed, sex and the presence of recurrent seizures (i.e. epilepsy) was found in our study population (Table 1). As expected, horses exhibiting chronic neurological signs $(>48 \mathrm{~h}$ ) were more likely to suffer from epilepsy compared with horses with acute neurological signs. Horses with seizures provoked by an identified factor (i.e. head trauma) were less likely to have epilepsy. Horses with epilepsy were less likely to exhibit seizure episode during hospitalisation. In addition, horses exhibiting abnormal mentation were less likely to have epilepsy compared with horses without this primary complaint. Similarly, for a horse exhibiting cranial nerve or gait deficits, the odds of having epilepsy was significantly lower compared with a horse without these clinical findings. Horses with abnormal blood biochemistry profile were 4.5 times less likely to have epilepsy. For a horse with recording of focal epileptiform activity on EEG, the odds of having epilepsy were significantly increased compared with those for a horse without this finding on EEG. In contrast, for a horse with abnormal CT imaging of the head, the odds of having epilepsy were lower compared with a horse without these clinical findings.

Overall, the initial screening identified 10 variables significantly $(P \leq 0.05)$ associated with epilepsy. As expected, frequency of seizures was a perfect predictor for epilepsy and as such this parameter was not included in the final logistic regression model. Although the variables 'EEG' and 'CT imaging' were significantly correlated with epilepsy in the univariate analysis, these variables were not included in the final model because both of these tests were not performed in the majority of cases. The remaining variables satisfied the criteria for inclusion in the multivariate logistic regression model: blood biochemistry profile, mentation impairment, cranial nerve and gait deficits, seizure during hospitalisation, duration of neurological signs, and type of seizures (Table 2). When adjusted for the effects of confounding factors in the final model, horses with generalised seizures were at reduced risk of having epilepsy compared with those with partial seizures. The Hosmer and Lemeshow goodness-of-fit test indicated that the model fits the data well $(P=0.58)$.

\section{Discussion}

Epilepsy is a condition characterised by an enduring predisposition to generate recurrent $(\geq 2)$ seizures and includes a variety of disorders reflecting underlying chronic brain dysfunction $[1,2]$. Epilepsy is classified as idiopathic (primary, genetic origin), symptomatic or cryptogenic (secondary) [3]. A standardised classification has numerous advantages, including the foundation for a coherent and systematic approach in the diagnosis and treatment of epilepsy, and for a common mode of communication among clinicians. Although there are numerous case reports of seizures in horses, to our knowledge this is the first attempt to classify seizures and epilepsy. In the current study, epilepsy was identified in $70 \%$ of our population, based on the occurrence of 2 or more seizures. Symptomatic epilepsies were identified in $36 \%$ of cases because of identifiable underlying chronic structural brain disorders. Intracranial tumours were identified in $12 \%(3 / 26)$ of the horses. In addition, cholesterol granuloma was found in 15\% (4/26) of the horses with symptomatic epilepsy, although a cause-effect relationship could not be established in these cases. Although cholesterol granuloma has been previously reported in horses with seizure disorders [19], its pathological significance might be underestimated and will require further study. Although EPM is a rare cause of seizures in horses [20], it was suspected to cause seizures in 5 cases and antiprotozoal treatment was associated with clinical improvement in 2 patients. In the current study, the majority of horses (54.8\%) exhibited recurrent seizures associated with unremarkable neurodiagnostic examinations and were classified as cryptogenic epilepsy. Cryptogenic epilepsies (Greek cryptos $=$ hidden) are suspected to be symptomatic, but the aetiology cannot be identified [3]. Therefore, the ILAE commission recently reclassified this type of epilepsy as 'unknown cause' [21], underlying the fact that establishment of such classification is a dynamic process. As in man, epilepsies of unknown cause account for the majority of all epilepsy [21], demonstrating the need for future research in neurodiagnostic testing and brain imaging. In addition, a complete diagnostic testing was not performed in the majority of our patients (e.g. lack of post mortem examination and/or in cases admitted prior to the acquisition of the CT facility), and the prevalence of symptomatic epilepsy may have been underestimated in this study. The prevalence of idiopathic epilepsy (also referred as 'genetic' in the most recent ILAE classification) was low in our study population, with 2 Arabian horses fulfilling the clinical requirements (diagnostic of exclusion with a suspected genetic aetiology), compared with report in other species $[4,8]$. The proportion of horses with epilepsy (including idiopathic) could have been underestimated in some animals reported with a single seizure episode when they were not well attended and/or when long-term follow-up was not available. However, in contrast with horses with recurrent seizures, the large majority of post mortem examinations in horses with single seizure revealed an immediate identified cause (e.g. calvarial fractures secondary to head trauma), rather than a chronic underlying disorder characteristic of epilepsy [2,7,17]. Finally, the prevalence of reactive seizures was low $(2 \%)$ in our study population. One could argue that this prevalence was also underestimated since some horses referred for obvious metabolic disturbances may not have been diagnosed or reported as having seizures, especially if they did not exhibit generalised seizures. Therefore, another limitation with the current study is its retrospective nature, since we relied on historical information from the medical records. However, we should point out that it has been demonstrated that seizure identification by clinical history is highly accurate (high sensitivity) in man [22], although similar study in horses is lacking.

The second objective of this study was to identify predicting factors in horses with recurrent seizures in order to help the clinician to diagnose epilepsy, an insidious disease. Indeed, as in other species [17], epileptic horses presented during interictus generally have a normal neurological 
TABLE 1: Results of univariate analysis of predictive factors for epilepsy $(n=104)$

\begin{tabular}{|c|c|c|c|c|c|c|}
\hline Variable & Category & $\begin{array}{l}\text { No. horses with } \\
\text { nonrecurrent seizure }\end{array}$ & $\begin{array}{l}\text { No. horses with } \\
\text { recurrent seizures }\end{array}$ & OR & $95 \% \mathrm{Cl}$ & $P$ value \\
\hline \multirow[t]{4}{*}{ Age } & $<1$ year & 4 & 5 & \multicolumn{2}{|c|}{ Reference } & NA \\
\hline & $1-4$ year & 8 & 9 & 0.9 & $0.2-4.6$ & 0.899 \\
\hline & 5-15 year & 14 & 41 & 2.34 & $0.6-9.97$ & 0.249 \\
\hline & >15 year & 5 & 18 & 2.88 & $0.6-14.9$ & 0.208 \\
\hline \multirow[t]{3}{*}{ Sex } & Gelding & 10 & 33 & \multicolumn{2}{|c|}{ Reference } & NA \\
\hline & Male & 8 & 11 & 0.42 & $0.1-1.3$ & 0.137 \\
\hline & Female & 13 & 29 & 0.68 & $0.3-1.8$ & 0.426 \\
\hline \multirow[t]{5}{*}{ Breed } & $\mathrm{QH}+$ related & 8 & 21 & \multicolumn{2}{|c|}{ Reference } & NA \\
\hline & $\mathrm{TB}$ & 6 & 17 & 1.08 & $0.3-3.7$ & 0.904 \\
\hline & Arabian & 9 & 11 & 0.47 & $0.1-1.6$ & 0.212 \\
\hline & Standardbred & 3 & 4 & 0.51 & $0.1-2.8$ & 0.436 \\
\hline & Other Breeds & 5 & 20 & 1.52 & $0.4-5.5$ & 0.517 \\
\hline \multirow[t]{3}{*}{$\mathrm{EEG}^{1}$} & Normal & 3 & 4 & \multicolumn{2}{|c|}{ Reference } & NA \\
\hline & Focal epileptic activity & 3 & 28 & 7 & $1.0-47.4$ & 0.046 \\
\hline & Abnormal; no focal epileptic activity & 2 & 12 & 4.5 & $0.5-37.4$ & 0.164 \\
\hline \multirow[t]{2}{*}{ CT head ${ }^{2}$} & Normal & 5 & 25 & \multicolumn{2}{|c|}{ Reference } & NA \\
\hline & Abnormal & 7 & 3 & 0.086 & $0.02-0.5$ & 0.004 \\
\hline \multirow[t]{2}{*}{ CSF analysis } & Normal & 13 & 46 & \multicolumn{2}{|c|}{ Reference } & NA \\
\hline & Abnormal & 8 & 17 & 0.61 & $0.2-1.7$ & 0.351 \\
\hline \multirow[t]{2}{*}{ EPM test (on CSF) ${ }^{3}$} & Normal & 4 & 9 & \multicolumn{2}{|c|}{ Reference } & NA \\
\hline & Abnormal & 1 & 4 & 1.78 & $0.2-21.4$ & 0.65 \\
\hline \multirow[t]{2}{*}{ Complete blood count } & Normal & 28 & 63 & \multicolumn{2}{|c|}{ Reference } & NA \\
\hline & Abnormal & 3 & 10 & 1.48 & $0.4-5.8$ & 0.573 \\
\hline \multirow[t]{2}{*}{ Blood biochemistry profile } & Normal & 22 & 67 & \multicolumn{2}{|c|}{ Reference } & NA \\
\hline & Abnormal & 9 & 6 & 0.22 & $0.1-0.7$ & 0.009 \\
\hline \multirow[t]{2}{*}{ Post mortem findings ${ }^{4}$} & Normal & 1 & 3 & \multicolumn{2}{|c|}{ Reference } & NA \\
\hline & Abnormal & 9 & 14 & 0.52 & $0.1-5.8$ & 0.594 \\
\hline \multirow[t]{3}{*}{ History of head trauma } & No & 22 & 52 & & rence & NA \\
\hline & Yes & 8 & 16 & 0.85 & $0.3-2.3$ & 0.739 \\
\hline & Suspected & 1 & 5 & 2.12 & $0.2-19.2$ & 0.505 \\
\hline Skull radiographs 5 & Normal & 4 & 14 & & rence & NA \\
\hline & Abnormal & 2 & 4 & 0.57 & $0.1-4.3$ & 0.589 \\
\hline Behaviour & Normal & 21 & 60 & & rence & NA \\
\hline & Abnormal & 10 & 13 & 0.46 & $0.2-1.2$ & 0.109 \\
\hline Mentation & Normal & 14 & 49 & & rence & NA \\
\hline & Abnormal & 17 & 24 & 0.4 & $0.2-1.0$ & 0.038 \\
\hline Cranial nerve deficits & Absent & 19 & 61 & & rence & NA \\
\hline & Present & 12 & 12 & 0.31 & $0.1-0.8$ & 0.016 \\
\hline Gait deficits & Absent & 6 & 31 & & rence & NA \\
\hline & Present & 25 & 42 & 0.33 & $0.1-0.9$ & 0.028 \\
\hline Provoking factors & Unknown & 25 & 70 & & rence & NA \\
\hline & Known & 6 & 3 & 0.12 & $0.1-0.8$ & 0.021 \\
\hline Seizure during hospital & No & 7 & 45 & & rence & NA \\
\hline & Yes & 24 & 28 & 0.18 & $0.1-0.5$ & 0.001 \\
\hline Neurological sign duration ${ }^{6}$ & $<48 h$ & 24 & 9 & & rence & NA \\
\hline & $>48 \mathrm{~h}$ & 7 & 62 & 23.62 & $7.91-70.56$ & $<0.001$ \\
\hline Type of seizure & Partial & 6 & 38 & & rence & NA \\
\hline & Generalised $^{7}$ & 25 & 35 & 0.19 & $0.1-0.5$ & 0.001 \\
\hline
\end{tabular}

$\mathrm{OR}=$ odds ratio; $\mathrm{EEG}=$ electroencephalography; $\mathrm{CT}=$ computed tomography; EPM = equine protozoal myeloencephalitis. Values in bold type were considered significant at $P<0.05$. NA: not applicable. 1 : Data were obtained for: ${ }^{1} 52$ horses; ${ }^{2} 40$ horses; ${ }^{3} 18$ horses; ${ }^{4} 27$ horses; ${ }^{5} 24$ horses; ${ }^{6} 102$ horses; ${ }^{7}$ included primarily and secondary generalised.

examination, since they were less likely to have abnormal mentation, and cranial nerve and/or gait deficits on admission compared with horses exhibiting a single episode (based on the univariate analysis). In addition, horses with epilepsy developed seizures unprovoked by any immediate identified factors and were less likely to develop seizures during hospitalisation. Therefore, the clinician rarely witnesses the seizure itself and the diagnosis of epilepsy is primarily based on a thorough history. Similar to meta-analysis studies of human epilepsy $[13,14,23]$ partial seizures were more prevalent in horses affected by epilepsy (based on the multivariate logistic regression). In addition, similar to reports in man and 
TABLE 2: Results of multivariate analysis of predictive factors for epilepsy

\begin{tabular}{|c|c|c|c|c|c|}
\hline Predictive factor $^{1}$ & Category & Wald Chi ${ }^{2}$ & Pvalue & OR & $95 \% \mathrm{Cl}$ \\
\hline \multirow[t]{2}{*}{ Blood biochemistry profile } & Normal & & & Reference & \\
\hline & Abnormal & 0.04 & 0.846 & 0.84 & $0.15-4.84$ \\
\hline \multirow[t]{2}{*}{ Mentation impairment } & Normal & & & Reference & \\
\hline & Abnormal & 0.71 & 0.398 & 2.02 & $0.40-10.32$ \\
\hline \multirow[t]{2}{*}{ Cranial nerve deficits } & No & & & Reference & \\
\hline & Yes & 0.47 & 0.491 & 0.56 & $0.11-2.88$ \\
\hline \multirow[t]{2}{*}{ Gait deficits } & Grade 0 & & & Reference & \\
\hline & Grade 1-5 & 0.06 & 0.801 & 0.82 & $0.18-3.74$ \\
\hline \multirow[t]{2}{*}{ Provocating factor } & Unknown & & & Reference & \\
\hline & Known & 0.92 & 0.338 & 0.32 & $0.03-3.30$ \\
\hline \multirow[t]{2}{*}{ Seizure during hospital } & No & & & Reference & \\
\hline & Yes & 0.13 & 0.721 & 0.77 & $0.18-3.23$ \\
\hline \multirow[t]{2}{*}{ Duration of neurological sign } & $<48 \mathrm{~h}$ & & & Reference & \\
\hline & $>48 \mathrm{~h}$ & 19.45 & $<0.0001$ & 27.70 & $6.33-121.21$ \\
\hline \multirow[t]{2}{*}{ Type of seizure } & Partial & & & Reference & \\
\hline & Generalised $^{1}$ & 7.00 & 0.008 & 0.14 & $0.03-0.60$ \\
\hline
\end{tabular}

Data were obtained for 102 horses. Values in bold type were considered significant at $\mathrm{P}<0.05$. 'included primarily and secondary generalised. Goodness-of-fit statistics: $\mathrm{R}^{2}: 0.41$, Max-rescaled R²: 0.58 , Hosmer and Lemeshow goodness-of-fit test: $\mathrm{Chi}^{2}=6.62$, degrees of freedom $=8$ and $\mathrm{P}=0.58$ (insignificant test indicates that the model fits the data well), c: 0.9 .

small animals, the presence of epileptiform paroxysmal activity on the EEG recording, which is defined as abnormal paroxysmal transient events (such as spikes, sharp waves, and spike-and-wave discharges), was strongly associated with recurrent seizures and thus supports the diagnosis of epilepsy $[12,14,24]$. Similarly, it has been reported that EEG has excellent sensitivity with a good specificity $(70 \%)$ to detect intracranial diseases in horses [15]. However, EEG findings do not exclude the diagnosis of epilepsy since normal interictal EEG recordings were reported in $11 \%$ of our cases exhibiting seizures. In contrast, the presence of epilepsy was significantly negatively associated with the presence of intracranial lesions detectable by $\mathrm{CT}$ imaging of the head based on univariate analysis. Except for subarachnoid haemorrhage and mineralised lesions, CT is less sensitive than magnetic resonance imaging (MRI) for the visualisation of soft tissue abnormalities in the equine brain $[4,16,25]$. However, CT scan is still a valuable diagnostic tool in cases of seizures secondary to head trauma (i.e. symptomatic seizures) and is warranted to rule out structural cerebral lesions as an aetiological diagnosis, especially if associated with other neurological signs such as cranial nerve deficits or altered mentation [16]. The results of the present study are consistent with reports in man, where EEG and MRI have mostly replaced the use of CT for the diagnosis of epilepsy [16]. However, since MRI equipment does not readily accommodate a mature equine head in most referral centres, the high incidence of cryptogenic (unknown) seizures reported in the present study could be in part explained by the current diagnostic limitations of the neuroimaging tools available in equine medicine. In addition, CT imaging and histopathological confirmation were not performed for all the cases, although the later also encounters diagnostic limitations since no brain/skull abnormalities were reported on post mortem examination in $15.7 \%(3 / 19)$ of horses with epilepsy $[15,25]$. As neuroimaging technology further progress in equine medicine, the prevalence of cryptogenic epilepsy may decrease in favour of symptomatic epilepsy.

A variety of terms have been used to describe equine seizure disorders and this study highlights the need for common agreement on the terminology of seizures and epilepsy among equine clinicians. This study is a first step in the definition and classification of seizures in an equine referral-based population. As in human neurology, such definitions are dynamic concepts and could help equine clinicians in the establishment of a diagnosis, a therapeutic plan and a prognosis for seizures.

\section{Authors' declaration of interests}

No conflicts of interest have been declared.

\section{Manufacturers' addresses}

${ }^{a}$ Grass, Amherst, Massachusetts, USA.

${ }^{\text {b}}$ Nihon-Kodon, Irvine, California, USA.

'Philips Medical Systems, Bothell, Washington, USA.

¿SAS, Cary, North Carolina, USA.

\section{References}

1. Engel, J. (2001) A proposal diagnostic scheme for people with epileptic seizures and with epilepsy: report of the ILAE Task Force on classification and terminology. ILAE Commission report. Epilepsia 42, 796-803.

2. Fisher, R.S., van Emde Boas, W., Blume, W., Elger, C., Genton, P., Lee, P. and Engel, J. (2005) Epileptic seizures and epilepsy: definitions proposed by the International League Against Epilepsy (ILAE) and the International Bureau for Epilepsy (IBE). Epilepsia 46, 470-472.

3. Anon (1989) Proposal for revised classification of epilepsies and epileptic syndromes. ILAE Commission on the classification and terminology of the International League Against Epilepsy. Epilepsia 30, 389-399.

4. Berendt, M. and Gram, L. (1999) Epilepsy and seizure classification in 63 dogs: a reappraisal of veterinary epilepsy terminology. J. Vet. Intern. Med. 13, 14-20.

5. Berendt, M., Gredal, H., Ersboll, A.K. and Alving, J. (2007) Premature death, risk factors, and life patterns in dogs with epilepsy. J. Vet. Intern. Med. 21, 754-759.

6. Podell, M., Fenner, W.R. and Powers, J.D. (1995) Seizure classification in dogs from a non referral-based population. J. Am. Vet. Med. Ass. 206, 1721-1728.

7. Podell, M. (2009) Differential diagnosis of seizures in dogs and cats. Proceedings of the Southern European Veterinary Conference, Barcelona.

8. Schriefl, S., Steinberg, T.A., Matiasek, K., Ossig, A., Fenske, N. and Fischer, A. (2008) Etiologic classification of seizures, signalment, clinical signs, and outcome in cats with seizure disorders: 91 cases (2000-2004). J. Am. Vet. Med. Ass. 233, 1591-1597.

9. Zimmerman, R., Hulsmeyer, V.I., Sauter-Louis, C. and Fischer, A. (2009) Status epilepticus and epileptic seizures in dogs. J. Vet. Intern. Med. 23, 970-976.

10. Aleman, M., Gray, L.C., Williams, D.C., Holliday, T.A., Madigan, J.E., LeCouteur, R.A. and Magdesian, K.G. (2006) Juvenile idiopathic epilepsy in Egyptian Arabian foals: 22 cases (1985-2005). J. Vet. Intern. Med. 20, 1443-1449.

11. Page, P., Parker, R., Harper, C., Guthrie, A. and Neser, J. (2006) Clinical, clinicopathologic, postmortem examination findings and familial history of 3 Arabians with lavender foal syndrome. J. Vet. Intern. Med. 20, 1491-1494.

12. Amatniek, J.C., Hauser, W.A., DelCastillo-Castaneda, C., Jacobs, D.M., Marder, K., Bell, K., Albert, M., Brandt, J. and Stern, Y. (2006) Incidence and predictors of seizures in patients with Alzheimer's disease. Epilepsia 47, 867-872. 
13. Kotsopoulos, I.A., van Merode, T., Kessels, F.G., de Krom, M.C. and Knottnerus, J.A. (2002) Systematic review and meta-analysis of incidence studies of epilepsy and unprovoked seizures. Epilepsia 43, 1402-1409.

14. Kotsopoulos, I., de Krom, M., Kessels, F., Lodder, J., Troost, J., Twellaar, M., van Merode, T. and Knottnerus, A. (2005) Incidence of epilepsy and predictive factors of epileptic and non-epileptic seizures. Seizure 14, 175-182.

15. Lacombe, V.A., Podell, M., Furr, M., Reed, S.M., Oglesbee, M.J., Hinchcliff, K.W. and Kohn, C.W. (2001) Diagnostic validity of electroencephalography in equine intracranial disorders. J. Vet. Intern. Med. 15, 385-393.

16. Sogaro-Robinson, C., Lacombe, V., Reed, S.M. and Balkrishnan, R. (2009) Factors predictive of abnormal results for computed tomography of the head in horses affected by neurologic disorders: 57 cases (2001-2007). J. Am. Vet. Med. Ass. 235, 176-183

17. Podell, M. (1996) Seizures in dogs. Vet. Clin. North. Am.: Small Anim. Pract. 26, 779-809.

18. Hayes, A.F. and Cai, L. (2007) Using heteroscedasticity-consistent standard error estimators in OLS regression: an introduction and software implementation. Behav. Res. Methods 37, 709-722.

19. Jackson, C.A., deLahunta, A., Dykes, N.L. and Divers, T.J. (1994) Neurological manifestation of cholesterinic granulomas in three horses. Vet. Rec. 135, 228-230.
20. Furr, M., MacKay, R., Granstrom, D., Schott II, H. and Andrews, F. (2002) Clinical diagnosis of equine protozoal myeloencephalitis (EPM). ACVIM consensus statement. J. Vet. Intern. Med. 16, 618-621.

21. Berg, A.T., Berkovic, S.F., Brodie, M.J., Buchhalter, J., Cross, J.H., van Emde Boas, W., Engel, J., French, J., Glauser, T.A., Mathern, G.W., Moshé, S.L., Nordli, D., Plouin, P. and Scheffer, I.E. (2010) Revised terminology and concepts for organization of seizures and epilepsies: report of the ILAE Commission on Classification and Terminology, 2005-2009. Epilepsia 51, 676-685.

22. Deacon, C., Wiebe, S., Blume, W.T., McLachlan, R.S., Young, G.B. and Matijevic, S. (2003) Seizure identification by clinical description in temporal lobe epilepsy: how accurate are we? Neurology 61, 1686-1689.

23. Forsgren, L., Bucht, G., Eriksson, S. and Bergmark, L. (1996) Incidence and clinical characterization of unprovoked seizures in adults: a prospective population-based study. Epilepsia 37, 224-229.

24. Holliday, T.A. and Williams, D.C. (1998) Interictal paroxysmal discharges in the electroencephalograms of epileptic dogs. Clin. Tech. Small Anim. Pract. 13, 132-143.

25. Lacombe, V.A., Sogaro-Robinson, C. and Reed, S.M. (2010) Diagnostic utility of computed tomography imaging in equine intracranial conditions. Equine Vet. J. 42, 393-399.

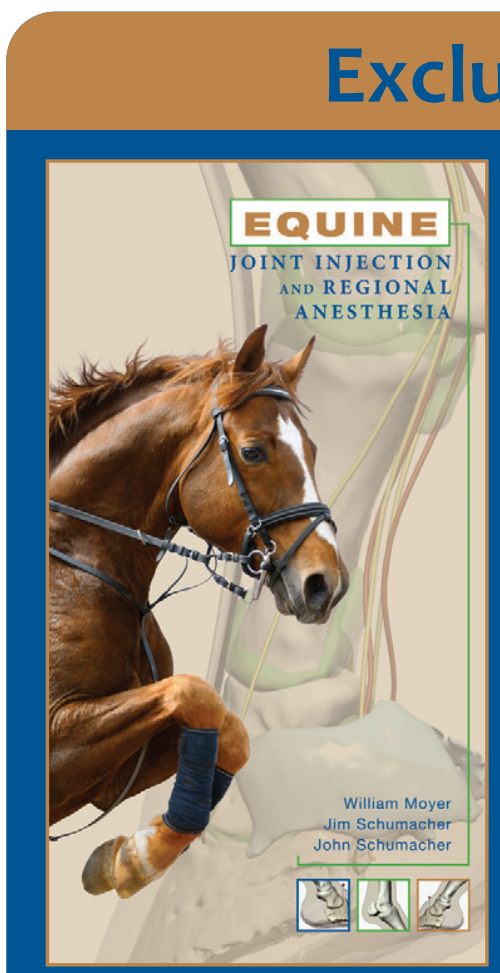

\title{
Equine Joint Injection and Regional Anesthesia
}

\author{
William Moyer, Jim Schumacher and John Schumacher \\ Publisher: Academic Veterinary Solutions \\ Hold your horses! \\ EVJ price: $£ 45.00$ plus p\&p \\ BEVA member price: $£ 40.50$
}

The new edition of Equine Joint Injection and Regional Anesthesia is A MUST-HAVE for every equine veterinarian! This comprehensive, easy-to-use resource is an invaluable tool to help you provide optimal care to your equine patients.

Written by three well-known experts, this updated, comprehensive book features expanded chapters, step-by-step photographs and all-new computer-generated images created by an equine anatomist to provide accurate, detailed depictions of equine anatomy. Equine Joint Injection and Regional Anesthesia remains the beloved, portable, userfriendly guide that is an invaluable tool for veterinarians working in all conditions, from the field to the referral hospital.

Order your copy today ....

EVJ Bookshop, Mulberry House, 31 Market Street, Fordham, Ely, Cambridgeshire CB7 5LQ, UK Tel: 01638723555 ^ Fax: 01638724043 ^ Email: bookshop@evj.co.uk ^ www.beva.org.uk 